\title{
Application and evaluation of a GPS multi-antenna system for dam deformation monitoring
}

\author{
Xiufeng $\mathrm{He}^{1}$, Guang Yang ${ }^{1}$, Xiaoli Ding ${ }^{2}$, and Yongqi Chen ${ }^{2}$ \\ ${ }^{1}$ College of Civil Engineering, Hohai University, Nanjing, China, 210098 \\ ${ }^{2}$ Department of Land Surveying and Geo-Informatics, the Hong Kong Polytechnic University, Hong Kong
}

(Received June 9, 2004; Revised October 13, 2004; Accepted November 1, 2004)

\begin{abstract}
This paper describes GPS multi-antenna device (one GPS receiver links multiple antennas) developed by authors, and the experimental results are presented. GPS has already proven to be an efficient tool for monitoring dam deformations and stability of high-risk slopes. It offers greater accuracy than other surveying techniques. However, GPS has its disadvantages when employing for slope and dam monitoring. The major drawback has been high cost due to large-scale GPS deployments are required in monitoring sites. The conventional GPS monitoring methods, where a permanent GPS receiver must be located at each point, have significant limitations of the cost. A new approach that a single GPS receiver links multiple antennas mounted at the monitoring points, has been employed to solve these problems in this paper. A dedicated switching device has been developed by authors for this approach. Field testing results show that the dedicated switching device for GPS multi-antenna system has excellent performances. Post-processing positioning accuracy is around 1-2 $\mathrm{mm}$ for the deformation monitoring of the Xiaolangdi dam on the Yellow River.
\end{abstract}

Key words: GPS, deformation monitoring, multi-antenna, switch, positioning.

\section{Introduction}

There are more than 22,000 dams in China. Further more, many steep slopes, both natural and engineered, exist near the dams. Most dams and engineered slopes were constructed in the 1950's and 1960's. Although it seems to be stable, the slopes near dams could slide due to some conditions such as heavy rain, flood and irrigation. Therefore there are potential problems of a dam health and safety, resulting in potential dam collapse that could threaten lives as well as properties. Several conventional techniques have been used to monitor the stability of slopes and dam safety. These include traditional surveying equipment and specialized geo-technical instruments. GPS is also employed as a monitoring tool for slopes and dams.

GPS has revolutionized positioning and surveying operation through providing accurate position and precise time globally and continuously. In addition, GPS has already proven to be an efficient tool for monitoring deformations of structures such as steep slopes, buildings, dams, and bridges (Ding et al., 2000; Forward, 1999; Goad, 1996). It offers greater accuracy than traditional surveying techniques (Ding et al., 2000; Forward, 1999). However, GPS has its disadvantages when employing for deformation monitoring. The major drawback has been high cost because largescale GPS deployments are required in monitoring sites. The conventional GPS monitoring methods, where a permanent GPS receiver must be located at each point to be monitored, thus have significant limitations. A new approach

Copy right(c) The Society of Geomagnetism and Earth, Planetary and Space Sciences (SGEPSS); The Seismological Society of Japan; The Volcanological Society of Japan; The Geodetic Society of Japan; The Japanese Society for Planetary Sciences; TERRAPUB. that a single GPS receiver links multiple antennas mounted at the monitoring point, has been proposed to solve these problems (Ding et al., 2000; Petrovski et al., 2000). The dedicated switching device for GPS multi-antenna switch (GMAS) has been developed by authors for this approach. By using the dedicated switching device, a GPS antenna is required for each point to be monitored, and a single GPS is employed for multiple monitoring points. Thus, the required hardware investments for deformations monitoring are significantly reduced, making GPS a much more using widely for continuously monitoring slope stability and dam safety.

This paper introduces the implementation of GMAS for GPS deformation monitoring system. The issues, such as the design and implementation of the RF switch, low-noise amplifiers for GPS signal and GMAS controller circuit have been discussed. The experimental results are given from the Xiaolangdi dam on the Yellow River.

\section{Multi-antenna System Description}

The fundamental requirement of any conventional GPS monitoring method is that a permanent GPS receiver must be located at each point to be monitored. Thus, the implementation of large-scale GPS deployments involves the high cost. To reduce the cost associated with the GPS deformation monitoring system, we have developed the GMAS. A single GPS receiver links multiple permanent antennae mounted at the monitoring point. Figure 1 shows the concept of a multi-antenna deformation monitoring system. Unlike the conventional GPS surveying method, eight antennae are connected to a single GPS receiver via the dedicated switching device. By switching antenna array in turn 


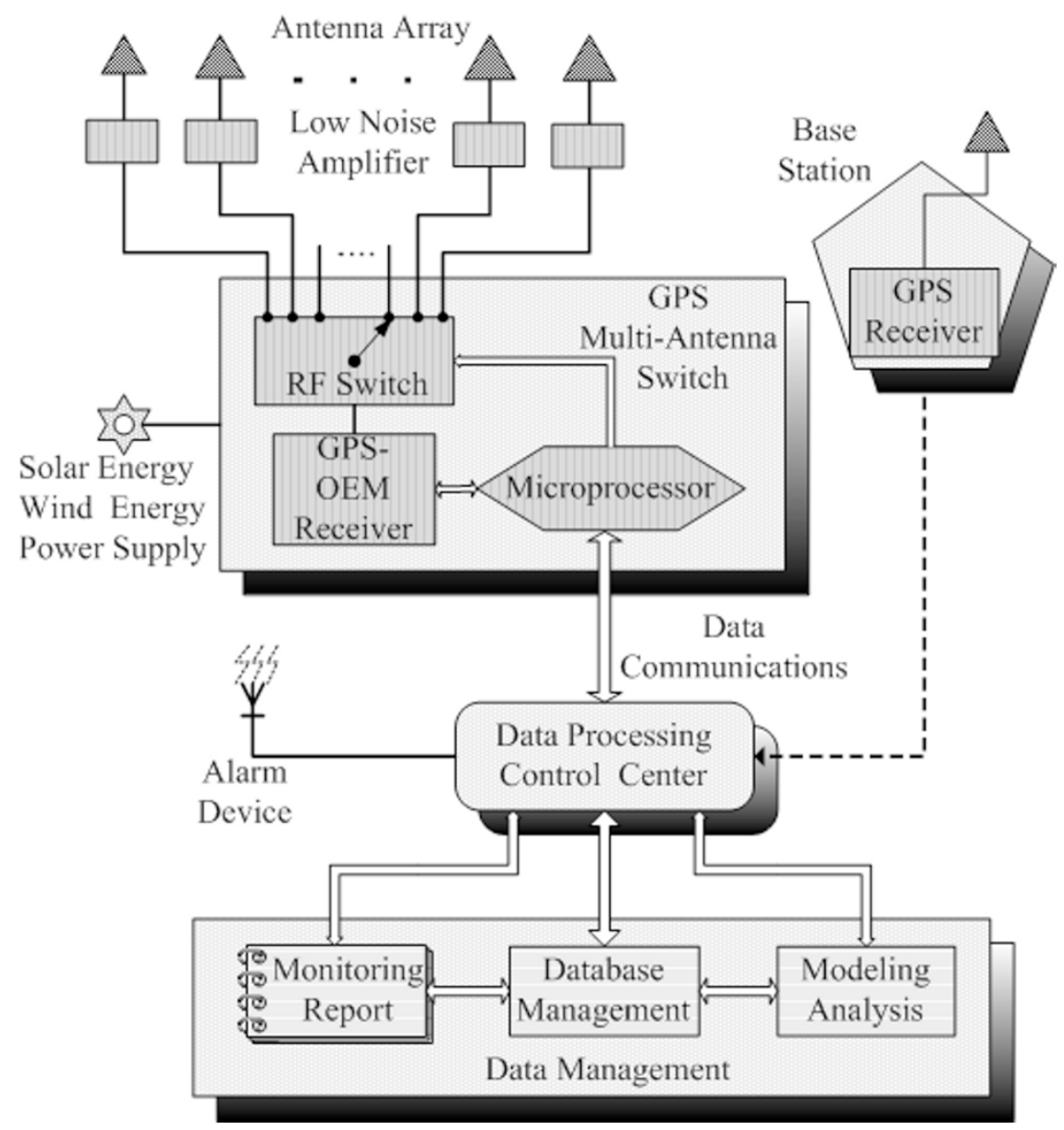

Fig. 1. The outline of multi-antenna GPS system for deformation monitoring.

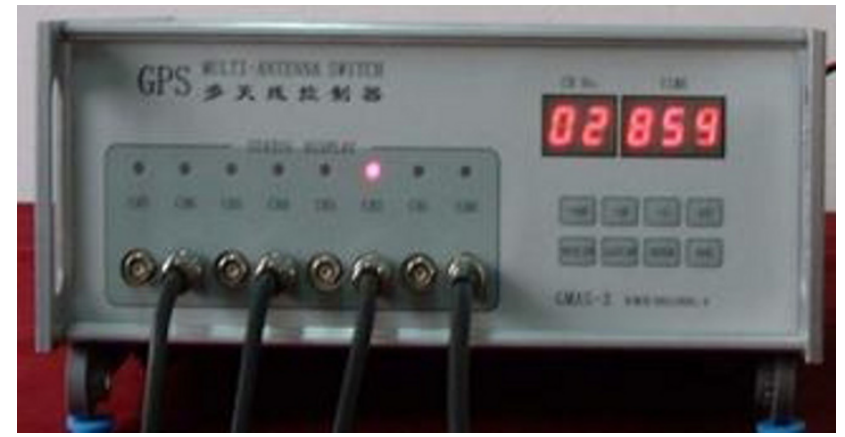

Fig. 2. GMAS prototype (Patented No: 00219891.6).

with interval of several minutes, the GPS receiver is employed to survey eight monitoring points. Under the control of the dedicated switching device, one GPS receiver could operate as eight receivers. Therefore, the costs of monitoring systems are reduced. As shown in Fig. 1, the base station GPS receiver is located at a stable site nearby. A communication link is used to transmit raw GPS observations to the data processing center.

\section{Antenna Switch Device}

As shown in Fig. 2, GMAS is a dedicated electronic switch designed for multi-antenna GPS deformation mon- itoring system. GMAS has been patented in 2002 in China (Patent No: 00219891.6, Owners: Xiufeng He, Xiaoli Ding, Yongrong Sun, Yongqi Chen et al.). Figure 3 shows the GMAS block diagram. The controller circuit has been designed to drive a radio-frequency (RF) switch together with microprocessor. The design of the circuit is illustrated in Fig. 4. GMAS selects an antenna sequentially under controlled by the microprocessor. The controller program is configured to support the switch circuit. The initial parameters such as the operating interval for GPS antennae can be entered from the keyboard into GMAS. Moreover, special commands are allowed to input from the keyboard into GMAS to change number of antennae. During monitoring period, GMAS operating status is displayed in two windows on the front of the equipment.

Specially designed RF switch is the key device for GMAS operation. RF switch was designed and implemented based on the dedicated 24 pins chip of microcircuits. It works in a fast efficient type of parallel processing architecture, and a matrix-form array is used to derive stable numerical algorithms suitable for parallel processing.

GMAS with different interface and connectors supports two modes for commercial GPS receivers. One can be allowed to directly connect with any standard survey-type GPS receivers and antennae. In another mode, GPS cards have been embedded into GMAS. We have tested successfully Ashtech GG24 dual frequencies GPS card and No- 


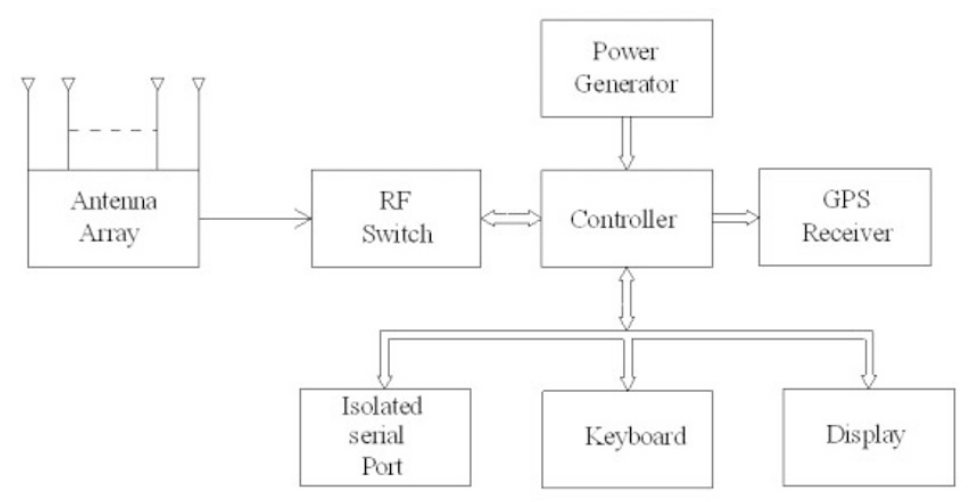

Fig. 3. GMAS block diagram.

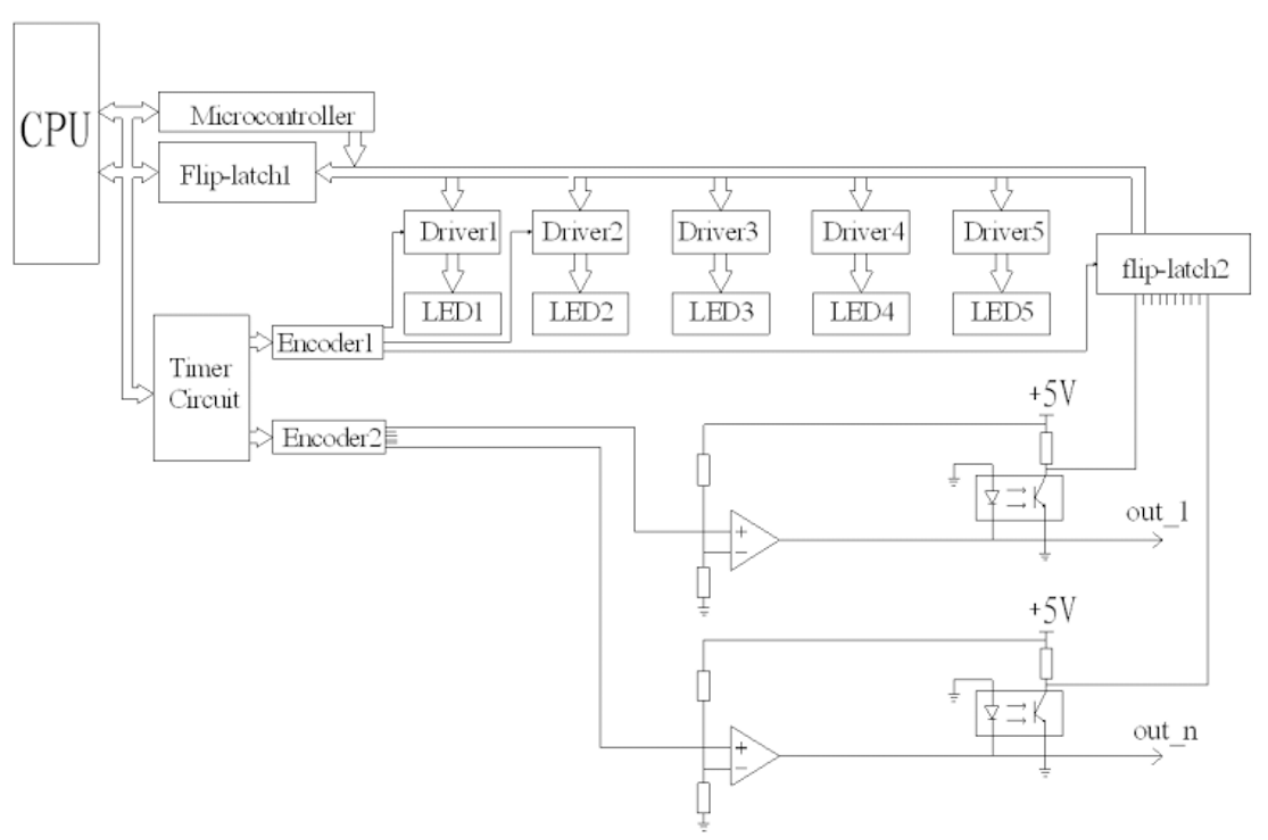

Fig. 4. GMAS circuit design.

vatel OEM4 dual frequencies GPS card. The GPS cards above are commercial GPS receivers, but the interface circuits have been designed and implemented by authors.

As a general requirement, the cable length, that is the distance between GPS antenna and GMAS, must be kept to less than 30 metres for coaxial-cable. In this case, GMAS cannot be used to monitor remotely and surveying area is limited significantly. From the views of engineering, we have developed the low-noise amplifiers for GMAS. The maximum distance between antenna and GMAS is now up to one kilometer. Tests have been successfully conducted using low-noise amplifiers for GMAS. Currently wireless communication device between GPS antenna and GMAS is under development. With such device, the cable lengths are free from the antenna to GMAS.

\section{Testing Results}

The experiments were firstly conducted in Hong Kong in 1999. A six-channel GMAS prototype was used to connect with two GPS antennas. The testing results have been given by Ding et al. (2000). These results show that GMAS was stable and precise positioning accuracy can be obtained. Further, the improved GMAS prototypes with eight-channel have been developed in 2001. They were used to monitor the deformation of slopes and dams in Hong Kong and mainland of China, respectively. In this paper, we present the results of the deformation monitoring for the Xiaolangdi dam on the Yellow River.

A variety of static experiments were conducted by GMAS using dual-frequencies GPS receivers to monitor the deformation of the Xiaolangdi dam in June 2002. There are four monitoring points, that is, 0003, 0019, 0021, and 0047. The control point 0004 is the base station. Sampling interval for GPS raw data was 20 seconds, and two hours GPS raw data were collected. Then double-differenced carrier phase measurements were used to resolve ambiguities, and the baseline vectors were calculated using the post processing software package developed by authors. The standard data from the traditional surveying techniques allow us to compare the experimental results of GMAS. 


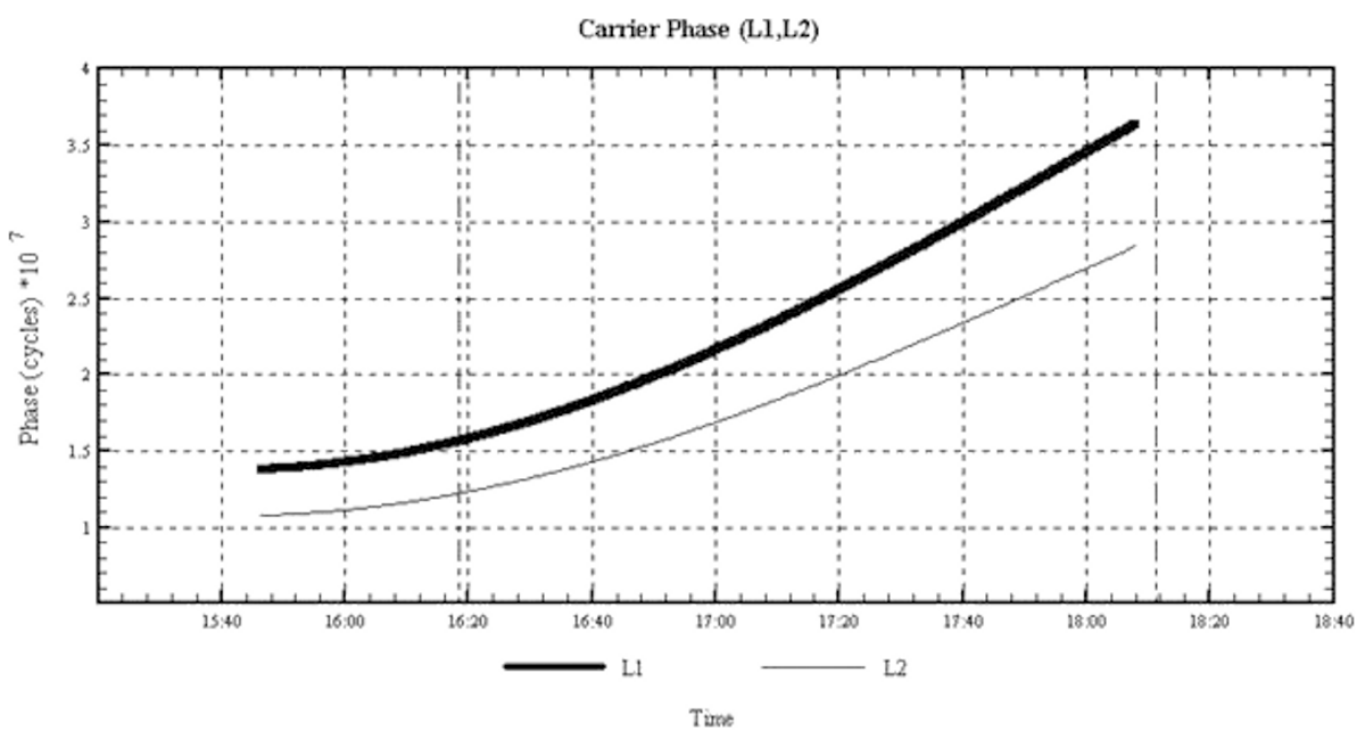

Fig. 5. GPS carrier phase measurements without GMAS.

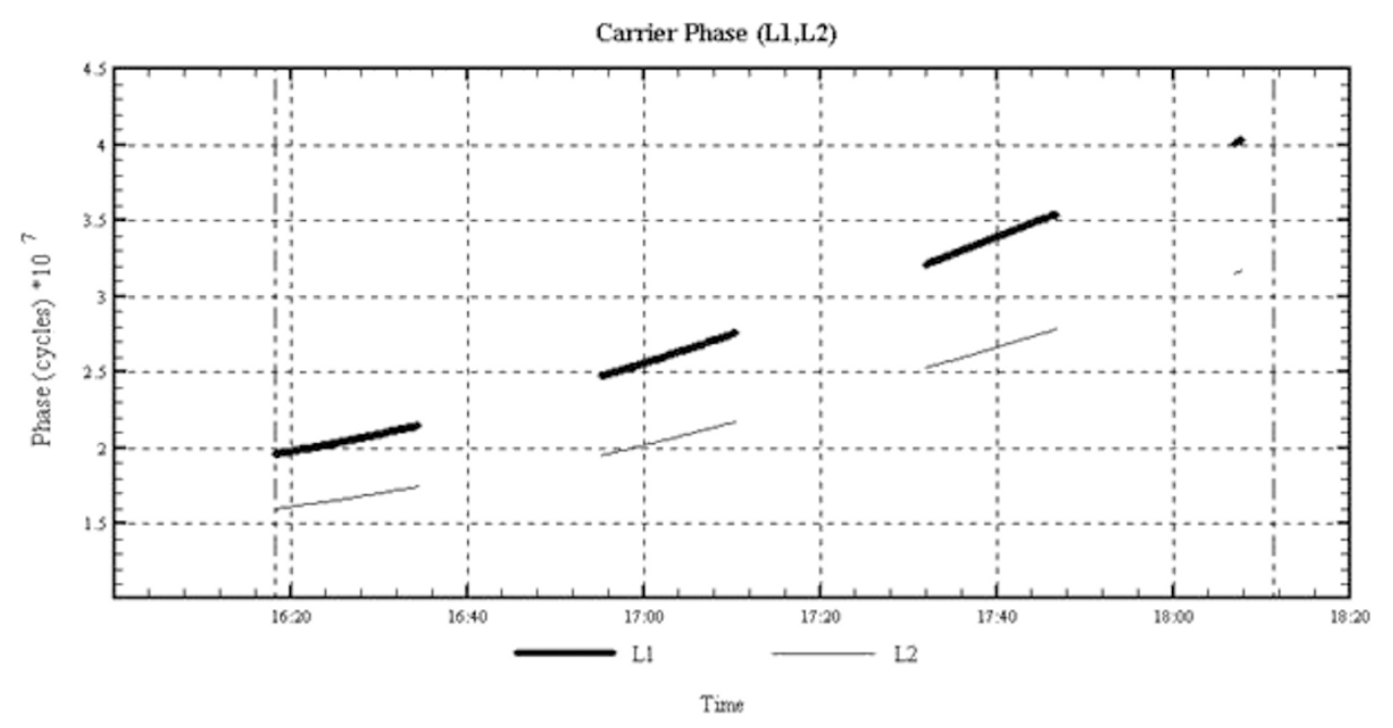

Fig. 6. GPS carrier phase measurements with GMAS.

Table 1. The results of baseline solutions.

\begin{tabular}{ccccc}
\hline Baseline & \multicolumn{4}{c}{ Multi-antenna method Conventional GPS } \\
\cline { 2 - 5 } $\begin{array}{c}\text { Vector } \\
(\mathrm{m})\end{array}$ & $\begin{array}{c}\text { Baseline } \\
(\mathrm{m})\end{array}$ & $\begin{array}{c}\text { RMS } \\
(\mathrm{m})\end{array}$ & $\begin{array}{c}\text { Baseline } \\
(\mathrm{m})\end{array}$ & RMS \\
\hline $0004-0047$ & 21.579 & 0.000 & 21.575 & 0.001 \\
$0004-0021$ & 37.515 & 0.001 & 37.515 & 0.001 \\
$0004-0003$ & 34.558 & 0.002 & 34.558 & 0.003 \\
$0004-0019$ & 143.375 & 0.001 & 143.373 & 0.002 \\
$0003-0019$ & 152.693 & 0.002 & 152.692 & 0.002 \\
$0021-0019$ & 120.180 & 0.001 & 120.178 & 0.001 \\
\hline
\end{tabular}

Figure 5 illustrates GPS (PRN 17) carrier phase measurements of the base station 0004 without GMAS. Figure 6 illustrates GPS (PRN 17) carrier phase measurements of the monitoring point 0047 with GMAS device. As GMAS device switched every fifteen minutes for each antenna, this resulted in the gap of the GPS phase observation in Fig. 6. Cycle slips occur whenever the GMAS switch from one antenna to the next antenna, and the cycle slips were repaired by post processing software package developed by authors. GMAS did not change the characteristics of GPS carrier phase. Thus, it had guaranteed that GPS data from different antennas with GMAS would be utilized for baseline solutions.

Table 1 illustrates the results of the baseline solutions from different baseline vectors. Four monitoring points were chosen to detect the length changes of six baselines. It can be seen from Table 1 that the errors of baseline lengths are $1-3 \mathrm{~mm}$ in conventional GPS method, while the errors are $1-2 \mathrm{~mm}$ in GPS multi-antenna method. Note that five survey-quality GPS receivers were required in conventional GPS network, however, one survey-quality GPS receiver was employed in GPS multi-antenna system. 


\section{Conclusions}

In this paper, we have introduced the GMAS and experimental results of the dam deformation monitoring. It can be seen from experimental results that post-processing positioning accuracy is around $1-2 \mathrm{~mm}$. The number of surveyquality receivers required in conventional GPS network has been reduced significantly. Thus, GPS multi-antenna system represents an economical and precise technique for deformation monitoring with a large-scale network. Especially the economic gain could increase rapidly with number of monitoring points.

It was also found in the tests that GPS multi-antenna system was stable and easy to set up and operate. This system is potential cost-effective solution for monitoring the deformation of constructed and natural structures such as dams, slopes and landslides. The remaining challenges are the implementation of wireless communication between GPS antenna and GMAS instead of coaxial cables presently. With such technique, the cable lengths are free from the GPS antenna to GMAS. Therefore, only GPS antennas are located in the points to be monitored.
Acknowledgments. The research work in this paper has been supported by National Nature Science Foundation of China, Grant No. 50279005 .

\section{References}

Ding, X. L., Y. Q. Chen, D. F. Huang, J. J. Zhu, M. Tsakiri, and M. Stewart, Slope Monitoring Using GPS: A Multi-antenna Approach, GPS World, 52-55, March, 2000.

Forward, T., Implementation Issues of an Integrated Satellite-Based Monitoring System for Open-pit Mine Walls, The 6th South East Asian Surveyors' Congress, Fremantle, Australia, November, 1999.

Goad, C., Surveying with the Global Positioning System, Chapter 18 in Global positioning system: Theory and application, Parkinson et al., American Institute of Aeronautics and Astronautics, Washington, DC, 1996.

Petrovski, I., S. Kawaguchi, H. Torimoto, M. Asako, T. Chachin, and K. Okano, LAMOS-BOHSAI: Landslide monitoring system based on high-speed sequential analysis for inclination, ION-GPS 2000, 84-94, 2000.

He, X., X. Ding, Y. Sun, Y. Chen, J. Liu, D. Huang, and J. Zhu, GPS Multiantenna switch, China Patent 00219891.6, 2002.

X. He (e-mail: gps@hhu.edu.cn), G. Yang, X. Ding, and Y. Chen 